\title{
A complete and strongly anonymous leximin relation on infinite streams
}

\section{Journal Article}

\section{Author(s):}

Asheim, Geir B.; Zuber, Stéphane

Publication date:

2013-10-01

Permanent link:

https://doi.org/10.3929/ethz-b-000071879

Rights / license:

In Copyright - Non-Commercial Use Permitted

Originally published in:

Social Choice and Welfare 41(4), https://doi.org/10.1007/s00355-012-0705-z 


\title{
A complete and strongly anonymous leximin relation on infinite streams
}

\author{
Geir B. Asheim • Stéphane Zuber
}

Received: 27 July 2011 / Accepted: 3 October 2012 / Published online: 13 October 2012

(C) Springer-Verlag Berlin Heidelberg 2012

\begin{abstract}
Various extensions of the leximin order to the infinite dimensional setting have been suggested. They relax completeness and strong anonymity. Instead, by removing sensitivity to generations at infinite rank this paper defines a complete and strongly anonymous leximin relation on infinite streams. The order is axiomatized, and it is shown to be the limit of extended rank-discounted utilitarianism for any utility function, as the discount factor approaches zero.
\end{abstract}

JEL Classification D63 - D71

\section{Introduction}

Maximin—which is often identified with Rawls's (1999) 'difference principle' and which in the intergenerational setting is the principle of giving extreme priority to the generation whose consumption has lowest rank-satisfies procedural equity: it treats generations equally by not being dependent on the sequence in which generations appear. However, it is only sensitive to the interests of the worst off generation.

G. B. Asheim

Department of Economics, University of Oslo, Oslo, Norway

e-mail: g.b.asheim@econ.uio.no

S. Zuber $(\varangle)$

CERSES, Université Paris Descartes and CNRS, Paris, France

e-mail: stephane.zuber@parisdescartes.fr

S. Zuber

Chair for Integrative Risk Management and Economics, Center for Economic Research, ETH Zürich,

Zurich, Switzerland 
In order to enhance sensitivity, Sen (1970) suggested maximin in its lexicographic form. This criterion, referred to as leximin, gives extreme priority to the generation whose consumption has lowest rank, while taking into account a generation whose consumption has higher rank if and only if there are ties at all lower ranks. In a finite setting, it is a complete, reflexive and transitive criterion which satisfies both procedural equity (by being indifferent to all permutations) and the strong Pareto principle (by being sensitive to each component of the consumption vector).

However, in a setting where an infinite, but countable, number of generations follow each other in sequence, it is impossible to combine the strong Pareto principle with full procedural equity (referred to as the axiom of strong anonymity), as shown by Van Liedekerke and Lauwers (1997). Moreover, any explicitly described complete, reflexive and transitive preferences satisfying the strong Pareto principle cannot even be combined with a weaker form of procedural equity (referred to as the axiom of finite anonymity) where the social evaluation is indifferent to only finite permutations; this is the Lauwers-Zame impossibility result (Lauwers 2010; Zame 2007).

To resolve the dilemma that arises due to the Lauwers-Zame impossibility result, completeness is usually dropped when leximin is extended to an infinite setting, while observing the strong Pareto principle and indifference to finite permutations (See Asheim 2010, Section 4, for an overview of this literature).

An alternative approach to the problem of extending leximin to an infinite setting is to start with maximin defined by the objective function $\inf _{t \in \mathbb{N}} x_{t}$, where $x_{t}$ is the consumption (indicating the wellbeing) of generation $t$. Recall that maximin is a complete, reflexive and transitive criterion satisfying the axiom of strong anonymity, even in the infinite setting. One can then ask how much sensitivity to the interests of any one generation can be introduced without producing a conflict with completeness and the axiom of strong anonymity. The present paper shows that the conflict arises only when trying to take into account generations whose consumption has infinite rank.

In the context of an extreme prioritarian criterion like leximin, one can argue that the interests of generations whose consumption has infinite rank should not matter, as for any such generation there are infinitely many generations that are worse off. Consequently, in the present paper we restrict the domain of the strong Pareto principle to finitely ranked generations, while retaining completeness and the axiom of strong anonymity. This produces a complete, reflexive and transitive leximin criterion which is fully procedurally equitable by satisfying the axiom of strong anonymity, but which does not take into account generations whose consumption has infinite rank.

The paper is organized as follows. In Sect. 2 we introduce the framework of our analysis. In Sect. 3 we define and provide an axiomatic foundation for the new leximin criterion for infinite consumption streams and discuss its properties. In Sect. 4 we show how the proposed leximin criterion is related to the extended rank-dependent utilitarian criterion recently introduced and analyzed by Zuber and Asheim (2012). In Sect. 5 we show how this new leximin criterion is related to other leximin criteria suggested in the infinite setting, while in Sect. 6 we offer concluding remarks. 


\section{The framework}

Let $\mathbb{N}$ denote as usual the set of natural numbers $\{1,2,3, \ldots\}$. Let $\mathbb{R}$ denote the set of real numbers, and $\mathbb{R}_{+}$the set of nonnegative real numbers.

Denote by $\mathbf{x}=\left(x_{1}, x_{2}, \ldots, x_{t}, \ldots\right)$ an infinite stream (or allocation), where $x_{t} \in$ $\mathbb{R}_{+}$is a one-dimensional indicator of the wellbeing of generation $t$. We refer to this indicator as the consumption of generation $t$, restrict attention to allocations consisting of bounded consumption streams, and denote by

$$
\mathbf{X}=\left\{\mathbf{x}=\left(x_{1}, \ldots, x_{t}, \ldots\right) \in \mathbb{R}_{+}^{\mathbb{N}}: \sup _{t} x_{t}<+\infty\right\}
$$

the set of possible allocations.

For $\mathbf{x}, \mathbf{y} \in \mathbf{X}$, write $\mathbf{x} \geq \mathbf{y}$ whenever $x_{t} \geq y_{t}$ for all $t \in \mathbb{N}$; write $\mathbf{x}>\mathbf{y}$ if $\mathbf{x} \geq \mathbf{y}$ and $\mathbf{x} \neq \mathbf{y}$; and write $\mathbf{x} \gg \mathbf{y}$ whenever $x_{t}>y_{t}$ for all $t \in \mathbb{N}$. For any $T \in \mathbb{N}$ and $\mathbf{x}, \mathbf{y} \in \mathbf{X}$, denote by $\mathbf{x}_{\mathbf{T}} \mathbf{y}$ the consumption stream $\mathbf{z}$ such that $z_{t}=x_{t}$ for all $t \leq T$ and $z_{t}=y_{t}$ for all $t>T$. For any $z \in \mathbb{R}_{+}$and $\mathbf{x} \in \mathbf{X}$, denote by $(z, \mathbf{x})$ the stream $\left(z, x_{1}, x_{2}, \ldots\right)$.

A permutation $\pi$ is a one-to-one map from $\mathbb{N}$ onto $\mathbb{N}$. For any $\mathbf{x} \in \mathbf{X}$ and permutation $\pi$, write $\mathbf{x}_{\pi}=\left(x_{\pi(1)}, x_{\pi(2)}, \ldots\right) \in \mathbf{X}$. Permutations can be represented by a permutation matrix, $P=\left(p_{i j}\right)_{i, j \in \mathbb{N}}$, which is an infinite matrix satisfying:

(1) For each $i \in \mathbb{N}, p_{i j(i)}=1$ for some $j(i) \in \mathbb{N}$ and $p_{i j}=0$ for all $j \neq j(i)$.

(2) For each $j \in \mathbb{N}, p_{i(j) j}=1$ for some $i(j) \in \mathbb{N}$ and $p_{i j}=0$ for all $i \neq i(j)$.

Given any permutation $\pi$, there is a permutation matrix $P$ such that, for any $\mathbf{x} \in$ $\mathbf{X}, \mathbf{x}_{\pi}=\left(x_{\pi(1)}, x_{\pi(2)}, \ldots\right)=P \mathbf{x}$. Conversely, given any permutation matrix $P$, there is a permutation $\pi$ defined by $\pi=P \mathbf{a}$, where $\mathbf{a}=(1,2,3, \ldots)$. The set of all permutations is denoted by $\mathcal{P}$.

A finite permutation $\pi$ is a permutation such that there is $T \in \mathbb{N}$ with $\pi(t)=t$ for all $t>T$. Thus, a finite permutation matrix has $p_{t t}=1$ for all $t>T$ for some $T \in \mathbb{N}$. The set of all finite permutations is denoted by $\mathcal{F}$.

Given a permutation matrix $P \in \mathcal{P}$ and $T \in \mathbb{N}$, we denote the $T \times T$ matrix $\left(p_{i j}\right)_{i, j \in\{1, \ldots, T\}}$ by $P(T)$. Let

$$
\begin{array}{r}
\mathcal{S}=\{P \in \mathcal{P}: \quad \text { there is some } k \in \mathbb{N} \text { such that, for each } T \in \mathbb{N}, \\
P(k T) \text { is a finite dimensional permutation matrix }\}
\end{array}
$$

denote the set of fixed-step permutations.

Two subsets of $\mathbf{X}$ will be of particular interest. First, we introduce the set $\mathbf{X}^{+}$of non-decreasing streams in $\mathbf{X}$. This set is defined as follows: $\mathbf{X}^{+}=\left\{\mathbf{x} \in \mathbf{X}: x_{t} \leq\right.$ $\left.x_{t+1}, \forall t \in \mathbb{N}\right\}$.

The second subset of $\mathbf{X}$, playing a key role in the remainder of the paper, is the set of allocations, $\overline{\mathbf{X}}$, whose elements can be permuted into non-decreasing streams. This set is defined as follows: $\overline{\mathbf{X}}=\left\{\mathbf{x} \in \mathbf{X}: \exists P \in \mathcal{P}, P \mathbf{x} \in \mathbf{X}^{+}\right\}$.

The following inclusions hold: $\mathbf{X}^{+} \subset \overline{\mathbf{X}} \subset \mathbf{X}$. In a finite setting, $\overline{\mathbf{X}}$ would be the same as $\mathbf{X}$. To see why this does not hold in an infinite setting, consider the stream 
$\mathbf{x}=(1,0,0, \ldots)$. For any permutation $\pi$, it must be that $\pi(1)<+\infty$ so that any reordered stream has the form $(0,0, \ldots, 0,1,0, \ldots)$. Hence, $\mathbf{x}$ cannot be reordered to form a non-decreasing stream.

To characterize the set $\overline{\mathbf{X}}$, let for each $\tau \in \mathbb{N}$ the function $\mathbf{r}_{\tau}: \mathbf{X} \rightarrow \mathbb{N} \cup\{\infty\}$ be defined by $\mathbf{r}_{\tau}(\mathbf{x})=\left|\left\{t \in \mathbb{N}: x_{t}<x_{\tau}\right\}\right|+1$ for all $\mathbf{x} \in \mathbf{X}$. Refer to $\mathbf{r}_{\tau}(\mathbf{x})$ as the rank of generation $\tau$, and say that generation $\tau$ 's rank is finite if $\mathbf{r}_{\tau}(\mathbf{x})<+\infty$.

Lemma 1 An allocation $\mathbf{x} \in \mathbf{X}$ belongs to $\overline{\mathbf{X}}$ if and only if, for all $t \in \mathbb{N}$, generation $t$ 's rank is finite.

Proof This is a restatement of Lemma 1 of Zuber and Asheim (2012).

Let the function $\ell: \mathbf{X} \rightarrow \mathbb{R}_{+}$be defined by $\ell(\mathbf{x})=\lim _{i n f} \operatorname{int}_{t \rightarrow} x_{t}$ for all $\mathbf{x} \in \mathbf{X}$; note that this function is well-defined since streams in $\mathbf{X}$ are bounded. By letting, for all $\mathbf{x} \in \mathbf{X}, L(\mathbf{x})$ denote $\left\{t \in \mathbb{N}: x_{t}<\ell(\mathbf{x})\right\}$, we obtain the following alternative characterization (Zuber and Asheim 2012, Proposition 1):

(a) If an allocation $\mathbf{x} \in \mathbf{X}$ satisfies $|L(\mathbf{x})|<+\infty$, then $\mathbf{x}$ belongs to $\overline{\mathbf{X}}$ if and only if $x_{t} \leq \ell(\mathbf{x})$ for all $t \in \mathbb{N}$.

(b) If an allocation $\mathbf{x} \in \mathbf{X}$ satisfies $|L(\mathbf{x})|=+\infty$, then $\mathbf{x}$ belongs to $\overline{\mathbf{X}}$ if and only if $x_{t}<\ell(\mathbf{x})$ for all $t \in \mathbb{N}$.

For $\mathbf{x} \in \overline{\mathbf{X}}$, denote by $\mathbf{x}_{[]}=\left(x_{[1]}, x_{[2]}, \ldots, x_{[r]}, \ldots\right)$ the non-decreasing infinite allocation which is a permutation of $\mathbf{x}$; i.e., there exists $P \in \mathcal{P}$ such that $\mathbf{x}_{[]}=P \mathbf{x} \in$ $\mathbf{X}^{+}$. Note that the permutation matrix $P$ need not be unique (for instance, if for some $t \neq t^{\prime}, x_{t}=x_{t^{\prime}}$, so that $\left.r_{t}(\mathbf{x})=r_{t^{\prime}}(\mathbf{x})\right)$, but the resulting non-decreasing allocation $\mathbf{x}_{[]}$is unique. In particular, it holds for all $t \in \mathbb{N}$ that $x_{\left[r_{t}(\mathbf{x})\right]}=x_{t}$. Likewise, for $\mathbf{x} \in \mathbf{X}$, denote by $\left(x_{[1]}, \ldots, x_{[|L(\mathbf{x})|]}\right)$ the non-decreasing finite allocation which is a permutation of the elements of $\mathbf{x}$ satisfying $t \in L(\mathbf{x})$.

A social welfare relation (SWR) on a set $\mathbf{X}$ is a binary relation $\succsim$, where for any $\mathbf{x}, \mathbf{y} \in \mathbf{X}, \mathbf{x} \succsim \mathbf{y}$ entails that the consumption stream $\mathbf{x}$ is deemed socially at least as good as $\mathbf{y}$. Let $\sim$ and $\succ$ denote the symmetric and asymmetric parts of $\succsim$. An SWR $\succsim^{\prime}$ is a subrelation to SWR $\succsim^{\prime \prime}$ if for all $\mathbf{x}, \mathbf{y} \in \mathbf{X}$, (a) $\mathbf{x} \sim^{\prime} \mathbf{y} \Rightarrow \mathbf{x} \sim^{\prime \prime} \mathbf{y}$ and (b) $\mathbf{x} \succ^{\prime} \mathbf{y} \Rightarrow \mathbf{x} \succ^{\prime \prime} \mathbf{y}$.

\section{Axiomatic foundation}

The difficulty of combining equal treatment of an infinite number of generations with sensitivity to the interests of each of these generations has been the topic of a prolific literature since the seminal contribution by Diamond (1965). Although complete social preferences over infinite streams that combine equal treatment with Paretian sensitivity exist (Svensson 1980), they cannot be explicitly described (Basu and Mitra 2003; Zame 2007; Lauwers 2010).

In this section we show how this impossibility is overcome by requiring only sensitivity to the interests of generations whose consumption has finite rank. In Subsect. 3.1 we first introduce the Pareto axiom restricted to non-decreasing streams together with other axioms used to characterize the leximin criterion we propose. In Subsect. 3.2 this 
criterion is then formally defined and fully characterized. In Subsect. 3.3 we discuss the properties of this criterion, in particular, that it is sensitive (only) to the interests of generations whose consumption has finite rank, and satisfies full procedural equity.

\subsection{Axioms}

Axiom $\mathbf{O}($ Order $)$ The SWR $\succsim$ is complete, reflexive and transitive on $\mathbf{X}$. An SWR satisfying axiom $\mathbf{O}$ is named a social welfare order (SWO).

Axiom M (Monotonicity) For any $\mathbf{x}, \mathbf{y} \in \mathbf{X}$, if $\mathbf{x}>\mathbf{y}$, then $\mathbf{x} \succsim \mathbf{y}$.

Axiom $\mathbf{M}$ is implied by the strong Pareto principle.

We then consider an axiom requiring sensitivity to the interests of each generation when comparing non-decreasing streams.

Axiom RSP (Restricted Strong Pareto) For any $\mathbf{x}, \mathbf{y} \in \mathbf{X}^{+}$, if $\mathbf{x}>\mathbf{y}$, then $\mathbf{x} \succ \mathbf{y}$.

We now turn to an axiom combining Koopmans' (1960) stationarity axiom (Postulate 4 ) with his separability Postulate $3 b$ (the axiom requiring that the evaluation of two streams with the same present consumption not depend on what that level of consumption is).

Axiom IF (Independent Future) For any $\mathbf{x}, \mathbf{y} \in \mathbf{X}$ and $z \in \mathbb{R}_{+},(z, \mathbf{x}) \succsim(z, \mathbf{y})$ if and only if $\mathbf{x} \succsim \mathbf{y}$.

If we extended our framework to also include comparisons at future times, then axiom IF would imply time consistency provided the SWR $\succsim$ is time invariant.

Next, we state two continuity axioms which have been weakened by restricting the domains of the streams $\mathbf{x}^{1}, \mathbf{x}^{2}, \ldots, \mathbf{x}^{k}, \ldots$ and the domain of the stream $\mathbf{x}$ to which this sequence converges to. Note that their use of the product topology is justified by means of prioritarianism for the worse-off because $\mathbf{x}$ is required to belong to $\mathbf{X}^{+}$.

Axiom RC1 (Restricted Continuity 1) For any $\mathbf{x} \in \mathbf{X}^{+}$and $\mathbf{y} \in \mathbf{X}$, if a sequence $\mathbf{x}^{1}, \mathbf{x}^{2}, \ldots, \mathbf{x}^{k}, \ldots$ of allocations in $\mathbf{X}$ is such that $\lim _{k \rightarrow \infty} \sum_{t \in \mathbb{N}}\left|x_{t}^{k}-x_{t}\right| 2^{-t}=0$ and, for each $k \in \mathbb{N}, x_{t}^{k} \in\left\{x_{t}, y_{t}\right\}$ for all $t \in \mathbb{N}$ and $\mathbf{x}^{k} \succsim \mathbf{y}\left(\operatorname{resp} . \mathbf{x}^{k} \precsim \mathbf{y}\right)$, then $\mathbf{x} \succsim \mathbf{y}$ (resp. $\mathbf{x} \precsim \mathbf{y}$ ).

Axiom RC2 (Restricted Continuity 2) For any $\mathbf{x} \in \mathbf{X}^{+}$and $\mathbf{y} \in \mathbf{X}$, if a sequence $\mathbf{x}^{1}, \mathbf{x}^{2}, \ldots, \mathbf{x}^{k}, \ldots$ of allocations in $\mathbf{X}$ is such that $\lim _{k \rightarrow \infty} \sum_{t \in \mathbb{N}}\left|x_{t}^{k}-x_{t}\right| 2^{-t}=0$ and, for each $k \in \mathbb{N}$, there exists $P_{k} \in \mathcal{P}$ satisfying $\mathbf{x}^{k} \geq P_{k} \mathbf{y}$ (resp. $\mathbf{x}^{k} \leq P_{k} \mathbf{y}$ ), then $\mathbf{x} \succsim \mathbf{y}(\operatorname{resp} . \mathbf{x} \precsim \mathbf{y})$.

Imposed continuity axioms must be restricted since, of course, an unrestricted continuity axiom is not compatible with leximin. Axiom RC1 is clearly weaker than unrestricted continuity (as only binary consumption choices are considered), while axiom RC2 is weaker than unrestricted continuity if $\succsim$ satisfies axioms M and SA since then $\mathbf{x}^{k} \geq P_{k} \mathbf{y}$ (resp. $\left.\mathbf{x}^{k} \leq P_{k} \mathbf{y}\right)$ implies $\mathbf{x}^{k} \succsim \mathbf{y}$ (resp. $\mathbf{x}^{k} \precsim \mathbf{y}$ ) while the converse implication does not hold. 
Finally, we state the strong axiom of procedural equity, requiring social indifference with respect to all permutation matrices $P \in \mathcal{P}$.

Axiom SA (Strong Anonymity) For any $P \in \mathcal{P}$ and $\mathbf{x} \in \mathbf{X}, \mathbf{x} \sim P \mathbf{x}$.

While the axiom of finite anonymity (i.e., anonymity in its weaker form, involving only finite permutations) is too weak - as it "only guarantees impartiality for a (negligibly) small part of the utility stream" (Van Liedekerke and Lauwers 1997, p. 165)-axiom SA might be too strong — as it produces hard-to-defend indifferences between streams (Lauwers 2011). We provide a discussion of the appropriateness of axiom SA in Subsect. 3.3.

\subsection{Characterization}

In this subsection we completely characterize the class of SWOs satisfying axioms $\mathbf{O}$, M, RSP, IF, RC1, RC2 and SA. As a first step, we do so within the restricted domain $\overline{\mathbf{X}}$ of streams that can be reordered into non-decreasing streams.

Definition 1 Strongly anonymous leximin SWO. An SWR $\succsim_{\mathcal{P}}^{L}$ on $\overline{\mathbf{X}}$ is a strongly anonymous leximin SWO (SAL SWO) if, for any $\mathbf{x}, \mathbf{y} \in \overline{\mathbf{X}}, \mathbf{x} \sim_{\mathcal{P}}^{L} \mathbf{y}$ if and only if $\mathbf{x}_{[]}=\mathbf{y}_{[]}$and $\mathbf{x} \succ_{\mathcal{P}}^{L} \mathbf{y}$ if and only if there exists $R \in \mathbb{N}$ such that $x_{[r]}=y_{[r]}$ for all $r \in\{1, \ldots, R-1\}$ and $x_{[R]}>y_{[R]}$.

Note that $\succsim_{\mathcal{P}}^{L}$ satisfies completeness and reflexivity on $\overline{\mathbf{X}}$ as, for any $\mathbf{x}, \mathbf{y} \in \overline{\mathbf{X}}, \mathbf{x}_{[]}=$ $\mathbf{y}_{[]}$or there exists $R \in \mathbb{N}$ such that $x_{[r]}=y_{[r]}$ for all $r \in\{1, \ldots, R-1\}$ and $x_{[R]} \neq y_{[R]}$. Showing that $\succsim_{\mathcal{P}}^{L}$ satisfies transitivity on $\overline{\mathbf{X}}$ is equally straightforward. Hence, $\succsim_{\mathcal{P}}^{L}$ is an order on $\overline{\mathbf{X}}$.

Proposition 1 If an $S W R \succsim$ on $\overline{\mathbf{X}}$ satisfies axioms $\boldsymbol{O}, \boldsymbol{R S P}, \boldsymbol{I F}, \boldsymbol{R C 1}$ and $\boldsymbol{S A}$, then it is an SAL SWO.

The proof of this result is based on the following intuition: Axiom RC1, with its use of the product topology combined with the requirement that the stream $\mathbf{x}$ to which $\mathbf{x}^{1}, \mathbf{x}^{2}, \ldots, \mathbf{x}^{k}, \ldots$ converges belong to $\mathbf{X}^{+}$, induces priority for a worse off generation. Axioms IF and SA imply that the priority for the worse off generation in conflicts between two generations cannot depend on the number of generations that have consumption levels in-between. This has as consequence that the priority must be extreme.

Proof of Proposition 1 Assume that $\mathbf{x}, \mathbf{y} \in \overline{\mathbf{X}}$ and that an SWR $\succsim$ on $\overline{\mathbf{X}}$ satisfies axioms $\mathbf{O}, \mathbf{R S P}, \mathbf{I F}, \mathbf{R C 1}$ and SA. Since $\succsim_{\mathcal{P}}^{L}$ is complete, it is sufficient to show that $\mathbf{x} \sim_{\mathcal{P}}^{L} \mathbf{y}$ implies $\mathbf{x} \sim \mathbf{y}$ and $\mathbf{x} \succ_{\mathcal{P}}^{L} \mathbf{y}$ implies $\mathbf{x} \succ \mathbf{y}$.

$\mathbf{x} \sim \sim_{\mathcal{P}}^{L} \mathbf{y}$ implies $\mathbf{x} \sim \mathbf{y}$. Assume $\mathbf{x} \sim_{\mathcal{P}}^{L} \mathbf{y}$. By Definition $1, \mathbf{x}_{[]}=\mathbf{y}_{[]}$. By $\mathbf{O}$ and $\mathbf{S A}$, $\mathbf{x} \sim \mathbf{y}$

$\mathbf{x} \succ_{\mathcal{P}}^{L} \mathbf{y}$ implies $\mathbf{x} \succ \mathbf{y}$. Assume $\mathbf{x} \succ_{\mathcal{P}}^{L} \mathbf{y}$. By Definition 1 , there exists $R \in \mathbb{N}$ such that $x_{[r]}=y_{[r]}$ for all $r \in\{1, \ldots, R-1\}$ and $x_{[R]}>y_{[R]}$. Construct $\tilde{\mathbf{x}}, \tilde{\mathbf{y}} \in \mathbf{X}^{+}$as follows: (i) $x_{[t]}=\tilde{x}_{t}=\tilde{y}_{t}=y_{[t]}$ for $t \in\{1, \ldots, R-1\}$, (ii) $x_{[R]}=\tilde{x}_{R}>\tilde{y}_{R}=y_{[R]}$, 
and (iii) $\tilde{x}_{t}=\tilde{x}=\inf _{r>R} x_{[r]}$ and $\tilde{y}_{t}=\tilde{y}=\sup _{r>R} y_{[r]}$ for $t>R$. It suffices to show $\tilde{\mathbf{x}} \succ \tilde{\mathbf{y}}$ as this implies $\mathbf{x} \succ \mathbf{y}$ by axioms O, RSP and SA.

In the case where $\tilde{x} \geq \tilde{y}$, then $\tilde{\mathbf{x}} \succ \tilde{\mathbf{y}}$ follows directly from axiom RSP. Hence, assume that $\tilde{x}<\tilde{y}$ and suppose $\tilde{\mathbf{x}} \precsim \tilde{\mathbf{y}}$. Consider the following sequence $\tilde{\mathbf{y}}^{1}, \tilde{\mathbf{y}}^{2}, \ldots$, $\tilde{\mathbf{y}}^{k}, \ldots$ of allocations in $\mathbf{X}$ :

$$
\tilde{y}_{t}^{k}= \begin{cases}\tilde{x} & \text { if } t=R+1, \ldots R+k \\ \tilde{y}_{t} & \text { otherwise }\end{cases}
$$

It follows from repeated application of axioms IF and SA that, for each $k \in \mathbb{N}, \tilde{\mathbf{x}} \precsim \tilde{\mathbf{y}}^{k}$. This in turn, by axiom RC1, implies that $\tilde{\mathbf{x}} \precsim \tilde{\mathbf{y}}^{\infty}$ where

$$
\tilde{y}_{t}^{\infty}= \begin{cases}\tilde{x} & \text { if } t>R \\ \tilde{y}_{t} & \text { otherwise }\end{cases}
$$

since $\tilde{\mathbf{y}}^{\infty} \in \mathbf{X}^{+}, \lim _{k \rightarrow \infty} \sum_{t \in \mathbb{N}}\left|\tilde{y}_{t}^{k}-\tilde{y}_{t}^{\infty}\right| 2^{-t}=0$, and for each $k \in \mathbb{N}, \tilde{y}_{t}^{k} \in\left\{\tilde{y}_{t}, \tilde{x}_{t}\right\}$ for all $t \in \mathbb{N}$. However, this contradicts that, by axiom RSP, $\tilde{\mathbf{x}} \succ \tilde{\mathbf{y}}^{\infty}$. Hence, $\tilde{\mathbf{x}} \succ \tilde{\mathbf{y}}$ also in the case where $\tilde{x}<\tilde{y}$.

A second step is to extend the SAL SWO to the entire domain $\mathbf{X}$ without conflict with any of the axioms $\mathbf{O}, \mathbf{M}, \mathbf{R S P}, \mathbf{I F}, \mathbf{R C 1}, \mathbf{R C 2}$ and SA. To do so, define, for any $\mathbf{x} \in \mathbf{X}, \overline{\mathbf{x}}$ as follows:

$$
\left\{\begin{array}{l}
\bar{x}_{t}=\min \left\{x_{t}, \ell(\mathbf{x})\right\} \text { for all } t \in \mathbb{N} \text { if }|L(\mathbf{x})|<+\infty \\
\overline{\mathbf{x}} \text { is the subsequence of } \mathbf{x} \text { consisting of all } x_{t} \text { with } t \in L(\mathbf{x}) \text { if }|L(\mathbf{x})|=+\infty
\end{array}\right.
$$

Lemma 1 implies that, by construction, $\overline{\mathbf{x}}$ belongs to $\overline{\mathbf{X}}$; therefore $\overline{\mathbf{x}}_{[]}$is well-defined.

Definition 2 Extended strongly anonymous leximin SWO. An SWR $\succsim_{\mathcal{P}}^{L}$ on $\mathbf{X}$ is an extended strongly anonymous leximin SWO (ESAL SWO) if, for any $\mathbf{x}, \mathbf{y} \in \mathbf{X}, \mathbf{x} \sim_{\mathcal{P}}^{L}$ $\mathbf{y}$ if and only if $\overline{\mathbf{x}}_{[]}=\overline{\mathbf{y}}_{[]}$and $\mathbf{x} \succ_{\mathcal{P}}^{L} \mathbf{y}$ if and only if there exists $R \in \mathbb{N}$ such that $\bar{x}_{[r]}=\bar{y}_{[r]}$ for all $r \in\{1, \ldots, R-1\}$ and $\bar{x}_{[R]}>\bar{y}_{[R]}$.

Proposition 2 The ESAL SWO $\succsim_{\mathcal{P}}^{L}$ on $\mathbf{X}$ satisfies axioms $\boldsymbol{O}, \boldsymbol{M}, \boldsymbol{R S P}, \boldsymbol{I F}, \boldsymbol{R C 1}, \boldsymbol{R C} 2$ and $\boldsymbol{S A}$.

Proof The ESAL SWO satisfies axiom $\boldsymbol{O}$. This follows from the facts that SAL SWO is an order and $\overline{\mathbf{x}}_{[]}$is well-defined for any $\mathbf{x} \in \mathbf{X}$.

The ESAL SWO satisfies axiom $\boldsymbol{M}$. Assume $\mathbf{x}>\mathbf{y}$ so that $\ell(\mathbf{x}) \geq \ell(\mathbf{y})$. Suppose $\mathbf{x} \prec_{\mathcal{P}}^{L} \mathbf{y}$, implying that there exists $R \in \mathbb{N}$ such that $\bar{x}_{[r]}=\bar{y}_{[r]}$ for all $r \in\{1, \ldots, R-1\}$ and $\bar{x}_{[R]}<\bar{y}_{[R]} \leq \ell(\mathbf{y}) \leq \ell(\mathbf{x})$. Hence,

$$
\left|\left\{t \in \mathbb{N}: x_{t}<\bar{y}_{[R]}\right\}\right| \geq R>R-1=\left|\left\{t \in \mathbb{N}: y_{t}<\bar{y}_{[R]}\right\}\right| .
$$

However, as $\mathbf{x}>\mathbf{y},\left\{t \in \mathbb{N}: x_{t}<\bar{y}_{[R]}\right\} \subseteq\left\{t \in \mathbb{N}: y_{t}<\bar{y}_{[R]}\right\}$, leading to a contradiction. Hence, by axiom $\mathbf{O}, \mathbf{x} \succsim_{\mathcal{P}}^{L} \mathbf{y}$. 
The ESAL SWO satisfies axiom RSP. The ESAL SWO corresponds to the SAL SWO on $\overline{\mathbf{X}} \supseteq \mathbf{X}^{+}$, and the SAL SWO satisfies axiom RSP.

The ESAL SWO satisfies axiom IF. Consider any $\mathbf{x}, \mathbf{y} \in \mathbf{X}$ and $z \in \mathbb{R}_{+}$, and write $\mathbf{x}^{\prime}=(z, \mathbf{x})$ and $\mathbf{y}^{\prime}=(z, \mathbf{y})$. Suppose $\mathbf{x} \prec_{\mathcal{P}}^{L} \mathbf{y}$, so that there exists $R \in \mathbb{N}$ such that $\bar{x}_{[r]}=\bar{y}_{[r]}$ for all $r \in\{1, \ldots, R-1\}$ and $\bar{x}_{[R]}<\bar{y}_{[R]}$. If $z \leq \bar{x}_{R}$, then $\bar{x}_{[r]}^{\prime}=\bar{y}_{[r]}^{\prime}$ for all $r \in\{1, \ldots, R\}$ and $\bar{x}_{[R+1]}^{\prime}<\bar{y}_{[R+1]}^{\prime}$. If $z>\bar{x}_{R}$, then $\bar{x}_{[r]}^{\prime}=\bar{y}_{[r]}^{\prime}$ for all $r \in\{1, \ldots, R-1\}$ and $\bar{x}_{[R]}^{\prime}<\min \left\{z, \bar{y}_{[R]}\right\}=\bar{y}_{[R]}^{\prime}$. In either case, $\mathbf{x}^{\prime} \prec_{\mathcal{P}}^{L} \mathbf{y}^{\prime}$, establishing by axiom $\mathbf{O}$ that $(z, \mathbf{x}) \succsim_{\mathcal{P}}^{L}(z, \mathbf{y})$ implies $\mathbf{x} \succsim_{\mathcal{P}}^{L} \mathbf{y}$. The converse follows likewise by showing that $(z, \mathbf{x}) \prec_{\mathcal{P}}^{L}(z, \mathbf{y})$ implies $\mathbf{x} \prec_{\mathcal{P}}^{L} \mathbf{y}$.

The ESAL SWO satisfies axiom $\mathbf{R C 1}$. Consider any $\mathbf{x} \in \mathbf{X}^{+}$and $\mathbf{y} \in \mathbf{X}$. Let the sequence $\mathbf{x}^{1}, \mathbf{x}^{2}, \ldots, \mathbf{x}^{k}, \ldots$ of allocations in $\mathbf{X}$ be such that, for each $k \in \mathbb{N}, x_{t}^{k} \in$ $\left\{x_{t}, y_{t}\right\}$ for all $t \in \mathbb{N}$ and $\mathbf{x}^{k} \succsim_{\mathcal{P}}^{L} \mathbf{y}$. Suppose $\mathbf{x} \prec_{\mathcal{P}}^{L} \mathbf{y}$, so that there exists $R \in \mathbb{N}$ such that $x_{r}=\bar{y}_{[r]}$ for all $r \in\{1, \ldots, R-1\}$ and $x_{R}<\bar{y}_{[R]}$. Since $\bar{y}_{[R]} \leq \ell(\mathbf{y}), \mid\{t \in$ $\left.\mathbb{N}: y_{t}<\bar{y}_{[R]}\right\} \mid=R-1$, implying since, for each $k \in \mathbb{N}, x_{t}^{k} \in\left\{x_{t}, y_{t}\right\}$ for all $t \in \mathbb{N}$ and $\mathbf{x}^{k} \succsim_{\mathcal{P}}^{L} \mathbf{y}$ that $\lim _{k \rightarrow \infty} \sum_{t \in \mathbb{N}}\left|x_{t}^{k}-x_{t}\right| 2^{-t} \geq\left|\bar{y}_{R}-x_{R}\right| 2^{-R}>0$. Hence, if $\lim _{k \rightarrow \infty} \sum_{t \in \mathbb{N}}\left|x_{t}^{k}-x_{t}\right| 2^{-t}=0$, then $\mathbf{x} \succsim_{\mathcal{P}}^{L} \mathbf{y}$, by axiom $\mathbf{O}$. Likewise if, for each $k \in \mathbb{N}, x_{t}^{k} \in\left\{x_{t}, y_{t}\right\}$ for all $t \in \mathbb{N}$ and $\mathbf{x}^{k} \precsim \precsim_{\mathcal{P}} \mathbf{y}$.

The ESAL SWO satisfies axiom $\boldsymbol{R C 2}$. Consider any $\mathbf{x} \in \mathbf{X}^{+}$and $\mathbf{y} \in \mathbf{X}$. Let the sequence $\mathbf{x}^{1}, \mathbf{x}^{2}, \ldots, \mathbf{x}^{k}, \ldots$ of allocations in $\mathbf{X}$ be such that, for each $k \in \mathbb{N}$, there exists $P_{k} \in \mathcal{P}$ satisfying $\mathbf{x}^{k} \geq P_{k} \mathbf{y}$. Suppose $\mathbf{x} \prec_{\mathcal{P}}^{L} \mathbf{y}$, so that there exists $R \in \mathbb{N}$ such that $x_{r}=\bar{y}_{[r]}$ for all $r \in\{1, \ldots, R-1\}$ and $x_{R}<\bar{y}_{[R]}$. Since $\bar{y}_{[R]} \leq \ell(\mathbf{y}), \mid\{t \in$ $\left.\mathbb{N}: y_{t}<\bar{y}_{[R]}\right\} \mid=R-1$, implying since, for each $k \in \mathbb{N}$, there exists $P_{k} \in \mathcal{P}$ satisfying $\mathbf{x}^{k} \geq P_{k} \mathbf{y}$ that $\lim _{k \rightarrow \infty} \sum_{t \in \mathbb{N}}\left|x_{t}^{k}-x_{t}\right| 2^{-t} \geq\left|\bar{y}_{R}-x_{R}\right| 2^{-R}>0$. Hence, if $\lim _{k \rightarrow \infty} \sum_{t \in \mathbb{N}}\left|x_{t}^{k}-x_{t}\right| 2^{-t}=0$, then $\mathbf{x} \succsim_{\mathcal{P}}^{L} \mathbf{y}$, by axiom $\mathbf{O}$. Likewise if there exists $P_{k} \in \mathcal{P}$ satisfying $\mathbf{x}^{k} \leq P_{k} \mathbf{y}$.

The ESAL SWO satisfies axiom $\mathbf{S A}$. For any $\mathbf{x} \in \mathbf{X}$, if there exists $P \in \mathcal{P}$ such that $\mathbf{y}=P \mathbf{x}$, then $\ell(\mathbf{y})=\ell(\mathbf{x})$ (as infinite permutations preserve lim inf) so that $\overline{\mathbf{x}}_{[]}=\overline{\mathbf{y}}_{[]}$ and $\mathbf{x} \sim \sim_{\mathcal{P}}^{L} \mathbf{y}$.

A third step is to show that the ESAL SWO is the only SWR satisfying axioms $\mathbf{O}, \mathbf{M}$, RSP, IF, RC1, RC2 and SA on the full domain X. This follows from the following proposition and is stated as the paper's main theorem.

Proposition 3 If an $S W O \succsim$ on $\mathbf{X}$ satisfies axioms $\boldsymbol{O}, \boldsymbol{M}, \boldsymbol{R C} 2$ and $\boldsymbol{S A}$, then $\overline{\mathbf{x}} \sim \mathbf{x}$ for any $\mathbf{x} \in \mathbf{X}$.

Proof Let $s(\mathbf{x})$ denote $\sup _{t \in \mathbb{N}} x_{t}$ for any $\mathbf{x} \in \mathbf{X}$.

Case 1: $|L(\mathbf{x})|<+\infty$. Since $\overline{\mathbf{x}} \leq \mathbf{x}$, it follows that $\overline{\mathbf{x}} \precsim \mathbf{x}$ by axioms $\mathbf{O}$ and $\mathbf{M}$. To show $\overline{\mathbf{x}} \succsim \mathbf{x}$, construct, for each $k \in \mathbb{N}, \overline{\mathbf{x}}^{k}$ as follows:

$$
\bar{x}_{t}^{k}= \begin{cases}\bar{x}_{[t]} & \text { if } t \leq|L(\mathbf{x})| \\ \ell(\mathbf{x})+\frac{1}{k}(s(\mathbf{x})-\ell(\mathbf{x})) & \text { if }|L(\mathbf{x})|+1 \leq t \leq|L(\mathbf{x})|+k \\ s(\mathbf{x}) & \text { if } t>|L(\mathbf{x})|+k .\end{cases}
$$


By Zuber and Asheim (2012, proof of Lemma 3), there exists, for each $k \in \mathbb{N}, P_{k} \in \mathcal{P}$ such that $\overline{\mathbf{x}}^{k} \geq P_{k} \mathbf{x}$. Since

$$
\sum_{t \in \mathbb{N}}\left|\bar{x}_{t}^{k}-\bar{x}_{[t]}\right| 2^{-t}=\left(\frac{1}{k}\left(1-2^{-k}\right)+2^{-k}\right)(s(\mathbf{x})-\ell(\mathbf{x})) 2^{-|L(\mathbf{x})|},
$$

we have that $\overline{\mathbf{x}}_{[]} \succsim \mathbf{x}$ by axiom RC2, and $\overline{\mathbf{x}} \succsim \mathbf{x}$ by axioms $\mathbf{O}$ and SA.

Case 2: $|L(\mathbf{x})|=+\infty$. Construct, for each $k \in \mathbb{N}, \overline{\mathbf{x}}^{k}$ as follows:

$$
\bar{x}_{t}^{k}= \begin{cases}\bar{x}_{[t]} & \text { if } t \leq k \\ s(\mathbf{x}) & \text { if } t>k\end{cases}
$$

Since there exists, for each $k \in \mathbb{N}, P_{k} \in \mathcal{P}$ such that $\overline{\mathbf{x}}^{k} \geq P_{k} \mathbf{x}$ and, furthermore, $\lim _{k \rightarrow \infty} \sum_{t \in \mathbb{N}}\left|\bar{x}_{t}^{k}-\bar{x}_{[t]}\right| 2^{-t}=0$, we have that $\overline{\mathbf{x}}_{[]} \succsim \mathbf{x}$ by axiom $\mathbf{R C 2}$, and $\overline{\mathbf{x}} \succsim \mathbf{x}$ by axioms $\mathbf{O}$ and $\mathbf{S A}$. Construct, for each $k \in \mathbb{N}, \mathbf{y}^{k}$ as follows:

$$
y_{t}^{k}= \begin{cases}\bar{x}_{[t]} & \text { if } t \leq k \\ \bar{x}_{[k]} & \text { if } t>k\end{cases}
$$

Since there exists, for each $k \in \mathbb{N}, P_{k} \in \mathcal{P}$ such that $\mathbf{y}^{k} \leq P_{k} \mathbf{x}$ and, furthermore, $\lim _{k \rightarrow \infty} \sum_{t \in \mathbb{N}}\left|y_{t}^{k}-\bar{x}_{[t]}\right| 2^{-t}=0$, we have that $\overline{\mathbf{x}}_{[]} \precsim \mathbf{x}$ by axiom $\mathbf{R C 2}$, and $\overline{\mathbf{x}} \precsim \mathbf{x}$ by axioms $\mathbf{O}$ and $\mathbf{S A}$. Hence, $\overline{\mathbf{x}} \sim \mathbf{x}$ also in this case.

Theorem 1 Consider an $S W R \succsim$ on $\mathbf{X}$. The following two statements are equivalent.

(1) $\succsim$ satisfies axioms $\boldsymbol{O}, \mathbf{M}, \boldsymbol{R S P}, \boldsymbol{I F}, \boldsymbol{R C 1}, \boldsymbol{R C 2}$ and $\boldsymbol{S A}$.

(2) $\succsim$ is an ESAL SWO.

Proof (1) implies (2). This follows from Propositions 1 and 3. (2) implies (1). This follows from Proposition 2.

\subsection{Properties}

We have alluded to the property that the ESAL SWO is sensitive to the interests of generations at finite rank. The following is a formal statement of this property.

Axiom SFR (Sensitivity to Finitely Ranked generations) For any $\mathbf{x}, \mathbf{y} \in \mathbf{X}$, if $\mathbf{x}>\mathbf{y}$ with $x_{\tau}>y_{\tau}$ for some $\tau \in \mathbb{N}$ satisfying $r_{\tau}(\mathbf{x}), r_{\tau}(\mathbf{y})<+\infty$, then $\mathbf{x} \succ \mathbf{y}$.

Axiom SFR implies axiom RSP as all generations have finite rank in a non-decreasing stream. Since all generations along a stream in $\overline{\mathbf{X}}$ have finite rank (by Lemma 1), it even implies that $\mathbf{x} \succ \mathbf{y}$ whenever $\mathbf{x}, \mathbf{y} \in \overline{\mathbf{X}}$ satisfy $\mathbf{x}>\mathbf{y}$.

Extreme priority of the worse off generation in conflicts between two generations is usually captured by the following axiom.

Axiom HE (Hammond Equity) For any $\mathbf{x}, \mathbf{y} \in \mathbf{X}$, if there exist $\tau, \tau^{\prime} \in \mathbb{N}$ such that $y_{\tau}<x_{\tau}<x_{\tau^{\prime}}<y_{\tau^{\prime}}$ and $y_{t}=x_{t}$ for all $t \neq \tau, \tau^{\prime}$, then $\mathbf{x} \succsim \mathbf{y}$.

The following result shows that the ESAL SWO satisfies these two axioms. 


\section{Proposition 4 The ESAL SWO $\succsim_{\mathcal{P}}^{L}$ on $\mathbf{X}$ satisfies axioms $\mathbf{S F R}$ and $\boldsymbol{H E}$.}

Proof The ESAL SWO satisfies axiom SFR. Consider $\mathbf{x}$ and $\mathbf{y}$ as in the definition of axiom SFR. The assumption that $r_{\tau}(\mathbf{x}), r_{\tau}(\mathbf{y})<+\infty$ implies that $x_{\tau} \leq \ell(\mathbf{x})$ and $y_{\tau} \leq \ell(\mathbf{y})$. Since $\mathbf{x}>\mathbf{y}$, we have that $\ell(\mathbf{x}) \geq \ell(\mathbf{y})$, and by the fact that the ESAL SWO satisfies axiom $\mathbf{M}$, we know that $\mathbf{x} \succsim_{\mathcal{P}}^{L} \mathbf{y}$.

Suppose $\mathbf{x} \sim_{\mathcal{P}}^{L} \mathbf{y}$ so that $\overline{\mathbf{x}}_{[]}=\overline{\mathbf{y}}_{[]}$by the definition of the ESAL SWO. Since $\lim _{r \rightarrow \infty} \bar{x}_{[r]}=\ell(\mathbf{x})$ and $\lim _{r \rightarrow \infty} \bar{y}_{[r]}=\ell(\mathbf{y})$, the case where $\ell(\mathbf{x})>\ell(\mathbf{y})$ would contradict $\overline{\mathbf{x}}_{[]}=\overline{\mathbf{y}}_{[]}$. Hence, consider the remaining case where $y_{\tau}<x_{\tau} \leq \ell(\mathbf{x})=\ell(\mathbf{y})$. Then there exists $R \in \mathbb{N}$ such that

$$
\left|\left\{t \in \mathbb{N}: x_{t} \leq y_{\tau}\right\}\right|<R=\left|\left\{t \in \mathbb{N}: y_{t} \leq y_{\tau}\right\}\right|
$$

Therefore, $x_{[R]}>y_{\tau}=y_{[R]}$, contradicting that $\overline{\mathbf{x}}_{[]}=\overline{\mathbf{y}}_{[]}$also in this case.

Thus, since $\mathbf{x} \succsim_{\mathcal{P}}^{L} \mathbf{y}$ and $\mathbf{x} \nsim_{\mathcal{P}}^{L} \mathbf{y}$, it follows that $\mathbf{x} \succ_{\mathcal{P}}^{L} \mathbf{x}$.

The ESAL SWO satisfies axiom HE. Consider $\mathbf{x}$ and $\mathbf{y}$ as in the definition of axiom HE. (a) If $\ell(\mathbf{x})=\ell(\mathbf{y}) \leq y_{\tau}$, then $\overline{\mathbf{x}}=\overline{\mathbf{y}}$ so that $\mathbf{x} \sim_{\mathcal{P}}^{L} \mathbf{y}$ by the definition of the ESAL SWO. (b) If $\ell(\mathbf{x})=\ell(\mathbf{y})>y_{\tau}$, let $R$ be the largest integer $r$ such that $\bar{y}_{[r]}=y_{\tau}$. By the definitions of $\overline{\mathbf{x}}_{[]}$and $\overline{\mathbf{y}}_{[]}$, it holds that $\bar{x}_{[r]}=\bar{y}_{[r]}$ for all $r \in\{1, \ldots, R-1\}$ and $\bar{x}_{[R]}>\bar{y}_{[R]}$ so that $\mathbf{x} \succ_{\mathcal{P}}^{L} \mathbf{y}$. Hence, in either case, $\mathbf{x} \succsim_{\mathcal{P}}^{L} \mathbf{x}$.

Since the ESAL SWO satisfies axiom SFR, this criterion is able to strictly rank some pairs of streams which are deemed equally good by maximin. To exemplify, consider starting with an egalitarian stream where all generations have the same consumption level $y$, and then compare this stream to the stream obtained by lifting a subset $N$ of the generations up to a higher consumption level $x$. By maximin the resulting stream is equally good as the original egalitarian stream even if only one generation remains at $y$. In contrast, by the ESAL SWO the new stream is strictly better as long as $N$ is cofinite, so that the set of generations remaining at $y$ is finite.

Such increased sensitivity may change the set of optimal streams in economic models. In particular, (Cairns and Tian, 2010, Section 4) claim that, for some set of initial conditions, there are efficient maximin streams in the Brander-Taylor model of the Easter Island (Brander and Taylor 1998) where individual wellbeing is non-decreasing and strictly increasing for a finite subinterval of time. For such initial conditions, keeping individual wellbeing constant at its initial maximin level is optimal (although not time-consistent) according to the maximin criterion, but not optimal according to the ESAL SWO.

As the ESAL SWO is not sensitive to the interests of generations at infinite rank, Theorem 1 demonstrates that sensitivity cannot be extended beyond finitely ranked generations under axioms O, M, RSP, IF, RC1, RC2 and SA. Hence, under these axioms the stream obtained by lifting a subset $N$ of the generations up to a higher consumption level $x$ is equally good as the original egalitarian stream if there are infinitely many generations remaining at $y$.

- On the one hand, given that the ESAL SWO satisfies the extreme form of prioritarianism that axiom HE captures, one may argue that it is reasonable that the criterion not be sensitive to the interests of generations at infinite rank. Because if 
generation $t$ is at infinite rank, there are infinitely many generations that are worse off. And by axiom HE, in conflicts between two generations, the one with the lower rank should have priority.

- On the other hand, the infinite number of generations remaining at $y$ might appear further and further apart so that the asymptotic density of the set of times $t$ with $x_{t}=y$ equals zero. In the context of an ordered set like time, this might be considered problematic.

This last observation is closely related to an argument set forward by Lauwers (2011), namely that the strong axiom of anonymity, SA, might be too strong when applied in the setting of an infinite and ordered set like time. In particular, consider two streams, $\mathbf{x}$ and $\mathbf{y}$, composed only of $x$ and $y$ (with $x>y$ as above), where $\liminf _{t \rightarrow+\infty} \mathbf{x}=\liminf _{t \rightarrow+\infty} \mathbf{y}=y$ and $\lim \sup _{t \rightarrow+\infty} \mathbf{x}=\lim \sup _{t \rightarrow+\infty} \mathbf{y}=x$. However, in stream $\mathbf{x}$, the $y$ s appear further and further apart, so that the asymptotic density of the set of positions $t$ with $x_{t}=y$ equals 0 , while in stream $\mathbf{y}$, the $x$ s appear further and further apart, so that the asymptotic density of the set of positions $t$ with $x_{t}=y$ equals 1 . By axiom $\mathbf{S A}$, these streams are equally good, which might be considered equally problematic.

In fact, axiom $\mathbf{S A}$ is even in conflict with the weak Pareto principle whereby one stream is preferred to another stream if the former has higher consumption than the latter at all times. This is demonstrated by the following adaptation of Fleurbaey and Michel's (2003) proof of their Theorem 1 to a setting where streams are bounded: Consider

$$
\begin{aligned}
& \mathbf{x}=\left(\frac{1}{3}, \frac{2}{3}, \frac{1}{4}, \frac{3}{4}, \ldots, \frac{1}{k+2}, \frac{k+1}{k+2}, \ldots\right) \\
& \mathbf{y}=\left(\frac{1}{4}, \frac{1}{3}, \frac{1}{5}, \frac{2}{3}, \ldots, \frac{1}{k+3}, \frac{k}{k+1}, \ldots\right),
\end{aligned}
$$

where by axiom SA $\mathbf{x}$ is indifferent to $\mathbf{y}$ even though $x_{t}>y_{t}$ for all $t \in \mathbb{N}$. Indeed, the ESAL SWO does not satisfy the weak Pareto principle (since $\mathbf{x} \sim{ }_{\mathcal{P}}^{L} \mathbf{y}$ whenever $\mathbf{x}$ and $\mathbf{y}$ satisfy $x_{t}>y_{t} \geq \ell(\mathbf{y})=\ell(\mathbf{x})$ for all $\left.t \in \mathbb{N}\right)$, and by the above demonstration this feature is necessary for an SWO satisfying axiom SA.

It must however be noticed that this conflict arises even between the weak Pareto principle and the axiom of finite anonymity. On the domain $[0,1]^{\mathbb{N}}$, Zame $(2007$, Th. 4) shows that any explicitly described complete, reflexive and transitive preferences cannot be proved to satisfy the weak Pareto principle and the axiom of finite anonymity. This result applies to our larger domain.

The present discussion illustrates the difficult nature of the dilemma posed by the Lauwers-Zame impossibility result. By restricting sensitivity to finitely ranked generations we have through the ESAL SWO been able to propose an explicitly described complete, reflexive and transitive SWR that satisfies procedural equity, not only in the sense of finite anonymity, but even in the form of axiom SA. It is an open and interesting question whether there exist versions of leximin where sensitivity is pushed beyond axiom SFR while retaining completeness and an appropriate form of procedural equity. 


\section{Limit of rank-discounted utilitarianism}

The extended rank-discounted utilitarian criterion is introduced and characterized by Zuber and Asheim (2012). This criterion coincides with discounted utilitarianism on the set of non-decreasing consumption streams. Utility discounting is then justified as an expression of inequality aversion when future generations are better off. However, and contrary to the discounted utilitarian approach, extended rank-discounted utilitarianism also satisfies procedural equity: two intergenerational consumption streams that are identical up to a permutation are deemed equally good. On streams that are not non-decreasing, discounting becomes the mere expression of intergenerational inequality aversion.

Definition 3 Extended Rank-Discounted Utilitarian SWO. An SWR on $\mathbf{X}$ is an Extended Rank-Discounted Utilitarian SWO (ERDU SWO) if it is represented by an SWF $W: \mathbf{X} \rightarrow \mathbb{R}$ defined by:

$$
W(\mathbf{x})=u(\ell(\mathbf{x}))+(1-\beta) \sum_{r=1}^{|L(\mathbf{x})|} \beta^{r-1}\left(u\left(x_{[r]}\right)-u(\ell(\mathbf{x}))\right)
$$

where $0<\beta<1$ is a real number and the function $u$ is continuous and increasing.

Write ${ }_{\beta, u}$ for the ERDU SWO characterized by $\beta$ and $u$. The following result establishes that, for any increasing and continuous function $u$, the ESAL SWO $\succsim_{\mathcal{P}}^{L}$ is the limit of the ERDU SWO $\succsim_{\beta, u}$ as $\beta$-the utility discount factor according to rank-approaches 0 .

Proposition 5 For any $\mathbf{x}, \mathbf{y} \in \mathbf{X}$ and any continuous and increasing function $u, \mathbf{x} \succsim_{\mathcal{P}}^{L}$ $\mathbf{y}$ if and only if there exists $\bar{\beta} \in(0,1)$ such that $\mathbf{x} \succsim_{\beta, u} \mathbf{y}$ for all $\beta \in(0, \bar{\beta})$.

Proof Assume that $\mathbf{x}, \mathbf{y} \in \overline{\mathbf{X}}$, and that $u$ is a continuous and increasing function. Since $\succsim_{\mathcal{P}}^{L}$ is complete, it is sufficient to show that $\mathbf{x} \sim_{\mathcal{P}}^{L} \mathbf{y}$ implies the existence of $\bar{\beta} \in(0,1)$ such that $\mathbf{x} \sim_{\beta, u} \mathbf{y}$ for all $\beta \in(0, \bar{\beta})$, and that $\mathbf{x} \succ_{\mathcal{P}}^{L} \mathbf{y}$ implies the existence of $\bar{\beta} \in(0,1)$ such that $\mathbf{x} \succ_{\beta, u} \mathbf{y}$ for all $\beta \in(0, \bar{\beta})$.

$\mathbf{x} \sim_{\mathcal{P}}^{L} \mathbf{y}$ implies the existence of $\bar{\beta} \in(0,1)$ such that $\mathbf{x} \sim_{\beta, u} \mathbf{y}$ for all $\beta \in(0, \bar{\beta})$. By Definition 2, $\overline{\mathbf{x}}_{[]}=\overline{\mathbf{y}}_{[]}$. By Definition 3, $\mathbf{x} \sim_{\beta, u} \mathbf{y}$ for all $\beta \in(0,1)$.

$\mathbf{x} \succ_{\mathcal{P}}^{L} \mathbf{y}$ implies the existence of $\bar{\beta} \in(0,1)$ such that $\mathbf{x} \succ_{\beta, u} \mathbf{y}$ for all $\beta \in(0, \bar{\beta})$. By Definition 2, there exists $R \in \mathbb{N}$ such that $\bar{x}_{[r]}=\bar{y}_{[r]}$ for all $r \in\{1, \ldots, R-1\}$ and $\bar{x}_{[R]}>\bar{y}_{[R]}$. Construct $\tilde{\mathbf{x}}, \tilde{\mathbf{y}} \in \mathbf{X}^{+}$as follows: (i) $\bar{x}_{[t]}=\tilde{x}_{t}=\tilde{y}_{t}=\bar{y}_{[t]}$ for $t \in\{1, \ldots, R-1\}$, (ii) $\bar{x}_{[R]}=\tilde{x}_{R}>\tilde{y}_{R}=\bar{y}_{[R]}$, and (iii) $\tilde{x}_{t}=\tilde{x}=\inf _{r>R} \bar{x}_{[r]}$ and $\tilde{y}_{t}=\tilde{y}=\sup _{r>T} \bar{y}_{[r]}$ for $t>T$. It suffices to show $\tilde{\mathbf{x}} \succ_{\beta, u} \tilde{\mathbf{y}}$ as this implies $\mathbf{x} \succ_{\beta, u} \mathbf{y}$ because $\succsim_{\beta, u}$ satisfies axioms $\mathbf{O}, \mathbf{M}$ and $\mathbf{S A}$.

In the case where $\tilde{x} \geq \tilde{y}$, then $\tilde{\mathbf{x}} \succ_{\beta, u} \tilde{\mathbf{y}}$ for all $\beta \in(0,1)$ follows directly because $\succsim_{\beta, u}$ satisfies axiom RSP. Hence, assume that $\tilde{x}<\tilde{y}$, and define $\bar{\beta}$ by

$$
(1-\bar{\beta}) u\left(\tilde{x}_{R}\right)+\bar{\beta} u(\tilde{x})=(1-\bar{\beta}) u\left(\tilde{y}_{R}\right)+\bar{\beta} u(\tilde{y}) .
$$

Then, by (1), $\tilde{\mathbf{x}} \sim_{\bar{\beta}, u} \tilde{\mathbf{y}}$, and $\tilde{\mathbf{x}} \succ_{\beta, u} \tilde{\mathbf{y}}$ for all $\beta \in(0, \bar{\beta})$. 
Proposition 5 bears some similarities to Basu and Mitra's (2007) robustness check for their utilitarian SWR (Basu and Mitra 2007, p. 361). ${ }^{1}$ Their idea is to start with the standard time-discounted utilitarian criterion and argue that the violation of procedural equity that the criterion entails is 'small' when the discount factor is close to one. They show that their utilitarian SWR orders any two streams the same way as time-discounted utilitarian criteria exhibiting appropriately 'small' violations of procedural equity.

In Proposition 5, we start from the ERDU SWO rather than the time-discounted utilitarian criterion, so that there is no violation of procedural equity, and we look at the case where the discount factor is close to zero. This means that we concentrate on cases where most priority is given to the poor. We show that our leximin criterion orders any two streams the same way as ERDU criteria exhibiting appropriately 'large' priority to the worst off.

\section{Comparison with other extensions of leximin}

In the recent literature on intertemporal social choice, leximin is usually defined in the setting of infinite consumption streams by extending the definition of leximin on the set of finite consumption streams to the infinite setting. To consider this literature and its relation to the present definition of leximin, let us reproduce the definition of leximin in the finite setting.

For this purpose, denote by $\mathbf{x}_{\mathbf{T}}$ the finite stream $\left(x_{1}, x_{2}, \ldots, x_{t}, \ldots, x_{T}\right)$ and denote by $\mathbf{x}_{[\mathbf{T}]}=\left(x_{[1]}, x_{[2]}, \ldots, x_{[r]}, \ldots, x_{[T]}\right)$ the non-decreasing allocation which is a permutation of $\mathbf{x}_{\mathbf{T}}$; i.e., there exists $P(T)$ such that $\mathbf{x}_{[\mathbf{T}]}=P(T) \mathbf{x}_{\mathbf{T}}$ is non-decreasing. Then $\succsim_{T}^{L}$ is defined by, for any $\mathbf{x}_{\mathbf{T}}, \mathbf{y}_{\mathbf{T}} \in \mathbb{R}_{+}^{T}, \mathbf{x}_{\mathbf{T}} \sim_{T}^{L} \mathbf{y}_{\mathbf{T}}$ if and only if $\mathbf{x}_{[\mathbf{T}]}=\mathbf{y}_{[\mathbf{T}]}$ and $\mathbf{x}_{\mathbf{T}} \succ_{T}^{L} \mathbf{y}_{\mathbf{T}}$ if and only if there exists $R \leq T$ such that $x_{[r]}=y_{[r]}$ for all $r \in$ $\{1, \ldots, R-1\}$ and $x_{[R]}>y_{[R]}$.

In the finite setting leximin satisfies both the strong Pareto principle and anonymity. However, in the infinite setting, as pointed out by Fleurbaey and Michel (2003), even the weak Pareto principle is in conflict with strong anonymity, i.e., axiom SA. Furthermore, Zame (2007, Th. 4) demonstrates that SWOs satisfying weak Pareto cannot be explicitly described even when finite anonymity (i.e., anonymity in its weaker form, involving only finite permutations) is imposed. Hence, when extending leximin to infinite streams in a manner that allows explicit description, one cannot keep both axioms $\mathbf{O}$ and $\mathbf{S A}$ while insisting on the strong Pareto principle.

In the present paper, we have kept axioms $\mathbf{O}$ and $\mathbf{S A}$ and weakened the strong Pareto principle, by requiring sensitivity only to generations at finite rank. Other explicitly describable extensions of leximin to the infinite setting have all weakened axiom $\mathbf{O}$. They can be divided in two parts depending on whether completeness or transitivity is relaxed.

Most of these extensions relax completeness. This is in particular the case of $\succsim^{L}$, under which streams are comparable only if they eventually coincide. The SWR $\succsim^{L}$ is defined as follows (cf. Asheim et al. 2010): For any $\mathbf{x}, \mathbf{y} \in \mathbf{X}, \mathbf{x} \succsim^{L} \mathbf{y}$ if and only if

\footnotetext{
1 We thank Kohei Kamaga for making this observation.
} 
there exists $T \in \mathbb{N}$ such that $\mathbf{x}_{\mathbf{T}} \succsim_{T}^{L} \mathbf{y}_{\mathbf{T}}$ and $\mathbf{x}=\mathbf{x}_{\mathbf{T}} \mathbf{y}$. This criterion does satisfy axiom SA, but fails the weak Pareto principle even for constant streams dominating each other. Bossert et al. (2007) suggest $\succsim_{\mathcal{F}}^{L}$, under which streams are comparable only if they eventually coincide or Pareto-dominates each other. The SWR $\succsim_{\mathcal{F}}^{L}$ is defined as follows: For any $\mathbf{x}, \mathbf{y} \in \mathbf{X}, \mathbf{x} \succsim_{\mathcal{F}}^{L} \mathbf{y}$ if and only if there exists $T \in \mathbb{N}$ such that $\mathbf{x}_{\mathbf{T}} \succsim_{T}^{L} \mathbf{y}_{\mathbf{T}}$ and $\mathbf{x} \geq \mathbf{x}_{\mathbf{T}} \mathbf{y}$. This criterion satisfies the strong Pareto principle and hence not axiom SA; rather, it satisfies finite anonymity.

Various other contributions show how comparability can be increased further by imposing anonymity involving fixed-step permutations (Lauwers 1997; Kamaga and Kojima 2009), different kinds of overtaking or catching-up (Asheim and Tungodden 2004; Asheim et al. 2010), or both (Asheim and Banerjee 2010; Kamaga and Kojima 2010). However, since these are explicitly described SWRs satisfying reflexivity, transitivity, finite anonymity and the strong Pareto principle, it follows from the results of Zame (2007) and Lauwers (2010) that they are not complete.

Given the incompleteness of the leximin criteria of the previous paragraph, one may ask whether they are subrelations to the complete leximin criterion $\succsim_{\mathcal{P}}^{L}$. This is not case since $(1,0,0,0, \ldots)$ is deemed socially better than $(0,0,0,0, \ldots)$ according to all of these incomplete criteria while they are equally good according to $\succsim_{\mathcal{P}}^{L}$ : $(1,0,0,0, \ldots) \sim_{\mathcal{P}}^{L}(0,0,0,0, \ldots)$.

In fact, there exist $\mathbf{x}, \mathbf{y} \in \mathbf{X}$ such that $\mathbf{x} \succ_{\mathcal{P}}^{L} \mathbf{y}$, while $\mathbf{x} \prec \mathbf{y}$ for any of different overtaking or catching-up leximin criteria $\succsim$ considered by Asheim and Tungodden (2004), Kamaga and Kojima (2010), Asheim et al. (2010) and Asheim and Banerjee (2010). Since the time-invariant overtaking leximin criterion $\succsim_{\mathcal{I}}^{L}$ of Asheim et al. (2010) is a subrelation to all the other overtaking or catching-up leximin criteria, this result is established through the following proposition.

Proposition 6 There exist $\mathbf{x}, \mathbf{y} \in \mathbf{X}$ such that $\mathbf{x} \succ_{\mathcal{P}}^{L} \mathbf{y}$ and $\mathbf{x} \prec_{\mathcal{I}}^{L} \mathbf{y}$.

To avoid the additional notation needed to define the time-invariant overtaking leximin criterion $\succsim_{\mathcal{I}}^{L}$, we state the following characterization.

Lemma 2 The following two statements are equivalent.

(1) $\mathbf{x} \succsim_{\mathcal{I}}^{L} \mathbf{y}$,

(2) For any $P \in \mathcal{P}$, there exists $T \in \mathbb{N}$ such that $(P \mathbf{x})_{\mathbf{T}} \succsim_{T}^{L}(P \mathbf{y})_{\mathbf{T}}$,

Proof (1) implies (2). Assume that (1) is true. As any $P \in \mathcal{P}$ maps a finite subset of $\mathbb{N}$ into another finite subset of $\mathbb{N}$, it follows directly from Asheim et al. (2010, Definition 4) that, for any $P \in \mathcal{P}$, there exists $T \in \mathbb{N}$ such that $(P \mathbf{x})_{\mathbf{T}} \succsim_{T}^{L}(P \mathbf{y})_{\mathbf{T}}$.

(2) implies (1). Suppose that (1) is not true. Then, by Asheim et al. (2010, the proof of Proposition 7), there exists $P \in \mathcal{P}$ such that $(P \mathbf{x})_{\mathbf{T}} \prec_{T}^{L}(P \mathbf{y})_{\mathbf{T}}$ for all $T \in \mathbb{N}$. This contradicts (2).

Proof of Proposition 6. Let $\mathbf{x}=(0,1,1, \ldots, 1, \ldots)$ and $\mathbf{y}=\left(1, \frac{1}{2}, \frac{1}{3}, \ldots, \frac{1}{n}, \ldots\right)$. Then $\bar{x}_{[1]}=0=\bar{y}_{[1]}$ and $\bar{x}_{[2]}=1>0=\bar{y}_{[2]}$ so that $\mathbf{x} \succ_{\mathcal{P}}^{L} \mathbf{y}$. We must show that $\mathbf{x} \prec_{\mathcal{I}}^{L} \mathbf{y}$; i.e., $\mathbf{x} \precsim_{\mathcal{I}}^{L} \mathbf{y}$, but not $\mathbf{x} \succsim_{\mathcal{I}}^{L} \mathbf{y}$. 
$\mathbf{x} \precsim \precsim_{\mathcal{I}}^{L} \mathbf{y}$ is true. Consider any $P \in \mathcal{P}$. Then there exists $\tau \in \mathbb{N}$ such that $\mathbf{x}_{\pi}=$ $\left(x_{\pi(1)}, x_{\pi(2)}, \ldots\right)=P \mathbf{x}$ satisfies $\pi(\tau)=1$ and $x_{\pi(\tau)}=0$. Choose $T \geq \tau$. Then $(P \mathbf{x})_{\mathbf{T}} \precsim_{T}^{L}(P \mathbf{y})_{\mathbf{T}}$. By Lemma 2, this implies $\mathbf{x} \precsim \precsim_{\mathcal{I}}^{L} \mathbf{y}$.

$\mathbf{x} \succsim_{\mathcal{I}}^{L} \mathbf{y}$ is not true. By Lemma 2 , it suffices to show the existence of $P \in \mathcal{P}$ such that $(P \mathbf{x})_{\mathbf{T}} \prec_{T}^{L}(P \mathbf{y})_{\mathbf{T}}$ for all $T \in \mathbb{N}$. Since $\mathbf{x}_{\mathbf{T}} \prec_{T}^{L} \mathbf{y}_{\mathbf{T}}$ for all $T \in \mathbb{N}$, this is obtained by setting $P$ equal to the identity matrix.

In his Theorem 5, Sakai (2010) considers variants of the leximin criteria satisfying completeness and the strong Pareto principle while failing transitivity. When comparing complete criteria, one SWR is a subrelation to another SWR if and only if they are identical. The leximin variants considered by Sakai (2010) are all different from $\succsim_{\mathcal{P}}^{L}$ as they, by the strong Pareto principle, deem $(1,0,0,0, \ldots)$ to be socially better than $(0,0,0,0, \ldots)$. Furthermore, it cannot be shown that $\mathbf{x} \succ_{\mathcal{P}}^{L} \mathbf{y}$ implies $\mathbf{x} \succ \mathbf{y}$ for the whole range of complete but intransitive leximin criteria $\succsim$ considered by Sakai (2010).

\section{Concluding remarks}

In this paper we have shown that the problem of combining completeness and procedural equity is resolved if one does not insist on sensitivity to the interests of generations whose consumption has infinite rank. In fact, then one can explicitly define a version of leximin over infinite consumption streams that is complete, reflexive and transitive, while satisfying the strong form of procedural equity that axiom SA entails. Provided that one adopts the extreme form of prioritarianism that leximin represents, perhaps it is reasonable not to care about generations who are better off than an infinite number of other generations.

It is important to note that the use of the product topology in axioms RC1 and RC2 does not reflect impatience, but priority for the worse-off, as the stream being approached is restricted to be non-decreasing. This entails that such prioritarianism need not be introduced by invoking axiom HE, which usually is employed to ensure extreme priority of the worse off generation in conflicts between two generations.

In the axiomatic foundations for discounted utilitarianism and extended rank-discounted utilitarianism, impatience (in the former case) and priority for the worse-off (in the latter case) are not introduced through the continuity axiom, as the uniform topology is used. Rather, impatience and priority for the worse-off respectively follow when such continuity is combined with versions of Koopmans' stationarity and separability conditions.

We have already shown that the ESAL SWO satisfies stationarity and separable future on the set of all streams (cf. axiom IF of Sect. 3.1), and it is straightforward to show that the criterion satisfies separable present on the set of non-decreasing streams. It is an open question whether introducing axiom HE, while weakening axioms RC1 and RC2 to versions where the uniform topology is used, is sufficient to characterize the ESAL SWO in view of these separability properties. 
Acknowledgements We thank Kohei Kamaga for pointing out an error in Proposition 2 of an earlier version, Wolfgang Buchholz for constructive suggestions concerning the proof of Proposition 4, Karl Schlag and Peter Vallentyne for discussions on the topic of this paper, and two anonymous referees, an associate editor and participants at conferences in Ancao (SAET) and Oslo (ESEM/EEA) and seminar presentations in Louvain-la-Neuve and Oslo for other helpful comments. This paper is part of the research activities at the Centre for the Study of Equality, Social Organization, and Performance (ESOP) at the Department of Economics at the University of Oslo. ESOP is supported by the Research Council of Norway. Asheim's research has also been supported by the Institut d'études avancées-Paris. Zuber's research has been supported by the Chair on Welfare Economics and Social Justice at the Institute for Global Studies (FMSH-Paris) and Franco-Swedish Program on Economics and Philosophy (FMSH \& Risksbankens Jubileumsfond).

\section{References}

Asheim GB (2010) Intergenerational equity. Annu Rev Econ 2:197-222

Asheim GB, d'Aspremont C, Banerjee K (2010) Generalized time-invariant overtaking. J Math Econ 46:519-533

Asheim GB, Banerjee K (2010) Fixed-step anonymous overtaking and catching-up. Int J Econ Theory 6:149-165

Asheim GB, Tungodden B (2004) Resolving distributional conflicts between generations. Econ Theory 24:221-230

Basu K, Mitra T (2003) Aggregating infinite utility streams with intergenerational equity: the impossibility of being Paretian. Econometrica 71:1557-1563

Basu K, Mitra T (2007) Utilitarianism for infinite utility streams: a new welfare criterion and its axiomatic characterization. J Econ Theory 133:350-373

Bossert W, Sprumont Y, Suzumura K (2007) Ordering infinite utility streams. J Econ Theory 135:579-589

Brander J, Taylor S (1998) The simple economics of Easter Island: a Ricardo-Malthus model of renewable resource use. Am Econ Rev 88:119-138

Cairns RD, Tian H (2010) Sustained development of a society with a renewable resource. J Econ Dyn Control 34:1048-1061

Diamond P (1965) The evaluation of infinite utility streams. Econometrica 33:170-177

Fleurbaey M, Michel P (2003) Intertemporal equity and the extension of the Ramsey criterion. J Math Econ 39:777-802

Kamaga K, Kojima T (2009) Q-anonymous social welfare relations on infinite utility streams. Soc Choice Welf 33:405-413

Kamaga K, Kojima T (2010) On the leximin and utilitarian overtaking criteria with extended anonymity. Soc Choice Welf 35:377-392

Koopmans TC (1960) Stationary ordinal utility and impatience. Econometrica 28:287-309

Lauwers L (1997) Infinite utility: insisting on strong monotonicity. Australas J Philos 75:222-233

Lauwers L (2010) Ordering infinite utility streams comes at the cost of a non-Ramsey set. J Math Econ 46:32-37

Lauwers L (2011) Strong anonymity and infinite streams, Discussion Paper 11.31, Center for Economic Studies, Katholieke Universiteit Leuven

Rawls J (1999) A theory of justice, revised edition. The Belknap Press of the Harvard University Press, Cambridge

Sakai T (2010) Intergenerational equity and an explicit construction of welfare criteria. Soc Choice Welf 35:393-414

Sen A (1970) Collective choice and social welfare. Holden-Day, San Francisco

Svensson L-G (1980) Equity among generations. Econometrica 48:1251-1256

Van Liedekerke L, Lauwers L (1997) Sacrificing the patrol: utilitarianism, future generations and infinity. Econ Philos 13:159-74

Zame WR (2007) Can intergenerational equity be operationalized?. Theor. Econ. 2:187-202

Zuber S, Asheim GB (2012) Justifying social discounting: the rank-discounted utilitarian approach. J Econ Theory 147:1572-1601 